\title{
Meteosat third generation infrared sounder (MTG-IRS), interferometer and spectrometer test outcomes, demonstration of the new 3D metrology system efficiency
}

Sylvain Abdon, Hubert Gardette, Cyril Degrelle, JeanMichel Gaucel, Patrick Astruc, et al.

Sylvain Abdon, Hubert Gardette, Cyril Degrelle, Jean-Michel Gaucel, Patrick Astruc, Patrice Guiard, Antonio Accettura, Daniel Lamarre, Donny M. Aminou, Didier Miras, "Meteosat third generation infrared sounder (MTG-IRS), interferometer and spectrometer test outcomes, demonstration of the new 3D metrology system efficiency," Proc. SPIE 11852, International Conference on Space Optics - ICSO 2020, 118521F (11 June 2021); doi:

$10.1117 / 12.2599240$

SPIE Event: International Conference on Space Optics - ICSO 2021, 2021, Online Only 


\section{International Conference on Space Optics-ICSO 2020}

Virtual Conference

30 March-2 April 2021

Edited by Bruno Cugny, Zoran Sodnik, and Nikos Karafolas
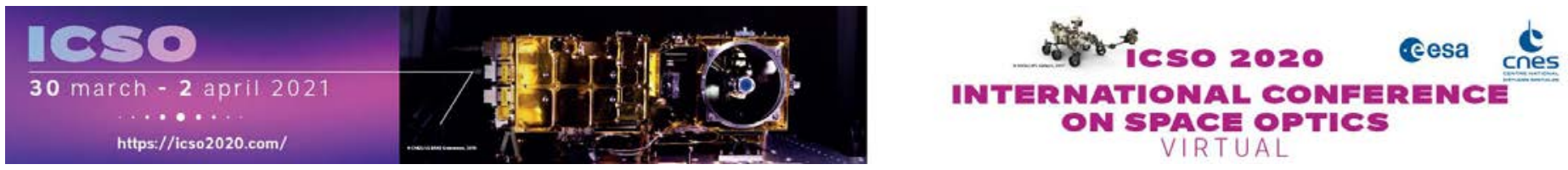

\section{Meteosat third generation infrared sounder (MTG-IRS), interferometer and spectrometer test outcomes, demonstration of the new 3D metrology system efficiency}

\section{Cesa issopocestings lenes}




\title{
Meteosat third generation infrared sounder (MTG-IRS), interferometer and spectrometer test outcomes, demonstration of the new 3D metrology system efficiency.
}

\author{
Sylvain Abdon ${ }^{\mathrm{a}}$, Hubert Gardette ${ }^{\mathrm{a}}$, Cyril Degrelle ${ }^{\mathrm{a}}$, Jean-Michel Gaucel ${ }^{\mathrm{a}}$, Patrick Astruc ${ }^{\mathrm{a}}$, \\ Patrice Guiard ${ }^{\mathrm{a}}$, Antonio Accettura ${ }^{\mathrm{b}}$, Daniel Lamarre ${ }^{\mathrm{c}}$, Donny Aminou ${ }^{\mathrm{c}}$, Didier Miras $^{\mathrm{a}}$ \\ ${ }^{\mathrm{a}}$ Thales Alenia Space - 5 allée des Gabians06156 Cannes La Bocca Cedex, France, ${ }^{\mathrm{b}}$ OHB System \\ AG, Manfred-Fuchs-Straße 182234 Weßling - Oberpfaffenhofen Germany, ${ }^{c}$ ESA - ESA/ESTEC, \\ Keplerlaan 1, PO Box 299, NL-2200 AG Noordwijk, The Netherlands
}

\begin{abstract}
During the last decade, meteorology domain has lived many breakthroughs. In the field of remote sensing from space, one of the most significant advancement is in the use of Thermal Infrared sounders. Thales Alenia Space delivered sounders flagship METOP/IASI, resulting in the enhancement of meteorological events forecast and unprecedented 3D atmospheric knowledge.

The next generation of European geostationary meteorological satellites, Meteosat Third Generation is currently being realized through the well-established and successful Cooperation between EUMETSAT and ESA with Thales Alenia Space being overall MTG prime contractor. MTG encompasses two kinds of satellites: an imaging mission MTG-I with the FCI imaging payload derived from the MSG-SEVIRI with a payload complement of a lightning detection system (LI as the Lightning Imager) and a sounding mission MTG-S with the payload complement including the thermal infrared sounding payload IRS (full disc coverage) and the Copernicus payload UVN/S4, a spectrometer in UV/VIS/NIR bands to retrieve air quality in Europe.

Stemming from IASI, Thales Alenia Space is now developing the IRS interferometer as well as the detection and onboard processing chain. These key elements will be integrated by OHB which is the IRS instrument as well as MTG-S satellite Prime Contractor. Reaching performance from a geostationary orbit is a two-order of magnitude more stringent challenge compared to a LEO instrument. Thales Alenia Space did elaborate new and innovative technology to ensure sub nanometric interferometer metrology and thus to achieve real time on-board corrections.
\end{abstract}

Two essential validation milestones have been achieved in 2019.

- The interferometer engineering model was thoroughly tested successfully, especially in terms of spectral performance vs. microvibrations.

- Thales Alenia Space has also tested the IRS "core spectrometer" engineering model, allowing to test the full spectrometer chain of the instrument by synchronizing the interferometer itself with the two 160x160 pixel detector arrays of the detection chain and the on-board data processing unit that is able to process the $2 \mathrm{Gbit} / \mathrm{s}$ raw data flow generated by the instrument.

The paper will focus on the two major outcomes of this test campaign:

- The demonstration of the functionality of IRS new 3D metrology system, its associated processing and its benefits.

- An overview of performances achieved during those two intensive test campaigns. In particular Spectral Response Function (SRF) knowledge/prediction, which is essential to support an unprecedented interpretation of the spectra and final products retrieval accuracy.

Keywords: Metosat, Fourier Transform Spectrometry, Spectro-imager. 


\section{INTRODUCTION}

In the frame of the European Space Agenency (ESA) Meteosat Third Generation (MTG) project, a new 3D metrology system has been implemented in the sounder instrument of the mission. The implementation of this new system is deemed necessary to achieve the MTG infrared sounder challenging specifications. This paper presents the validation strategy of this new technology and its outcomes.

\subsection{Metosat Third Generation Program}

The Metosat Third Generation mission involves three satellites flying in constellation in geostationary orbit above Europe (Figure 1):

- Two imager satellites MTG-I, one scanning the European area, the other scanning the full Earth disc

- The Flexible Combined Imager (FCI) provides high resolution images in 16 spectral band from InfraRed to Visible

- One sounding satellite MTG-S, scanning alternatively Europe and the rest of the Earth disc

○ The infra-red spectrometer (MTG-IRS) provides high resolution spectrograms in 2 spectral bands in InfraRed

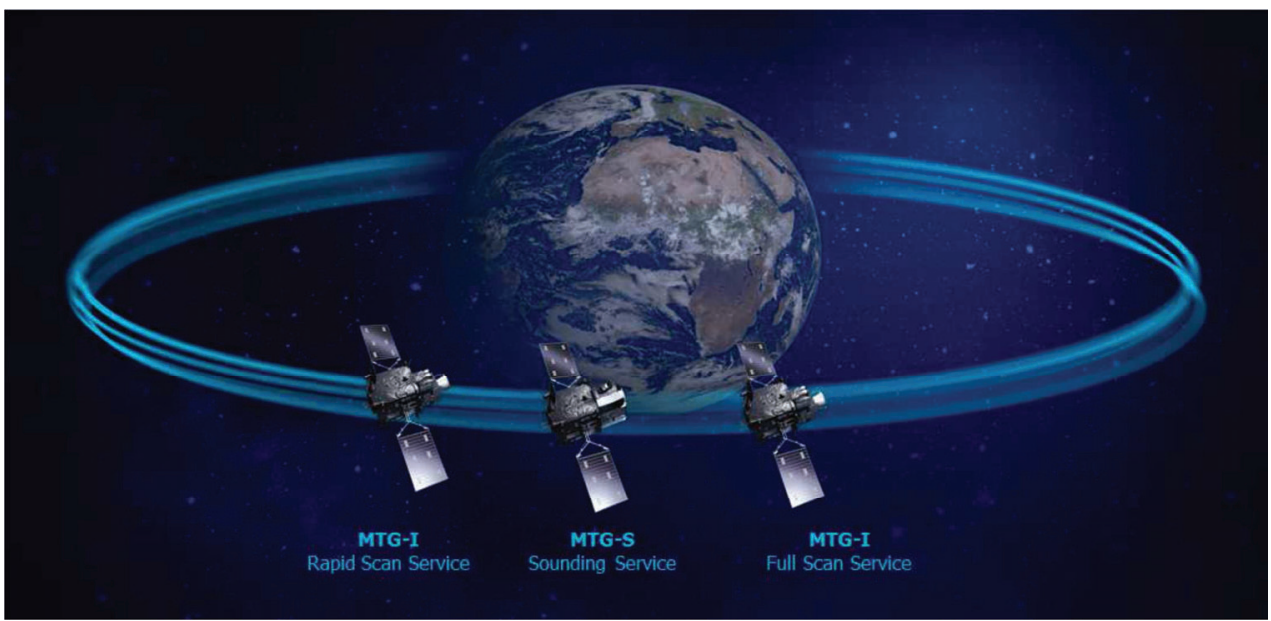

Figure 1: Metosat third generation mission involve a constellation of thee satellites.

MTG-IRS spectrometer is Fourier transform spectrometer (FTS) based on a Michelson Interferometer ([1], [2]). The theoretical background for this type of instrument is synthetized by Peter R. Griffiths, James A. de Haseth [3]. Demonstration of the interest of this kind of instrument for meteorology and atmosphere chemistry study was demonstrated with Infrared Atmospheric Sounding Interferometer (IASI) instrument, which is flying on Meteorological Operational satellite (Metop) [7].

The instrument is composed of four main sub-elements developed and manufactured in parallel (see Figure 2): The front telescope that scans the Earth and collects light from the Earth or from calibration scenes, the Michelson Interferometer Assembly, which is the core of the instrument, the back telescope that collects the flux of the interferometer and the detection and electronics assembly which contains optics, detection chain and signal processing units. The Core Spectrometer is a sub-assembly of the IRS. It is representative of the spectral behavior of the instrument. It was tested in a dedicated campaign using non-flying Engineering Models of the three involved subparts. 


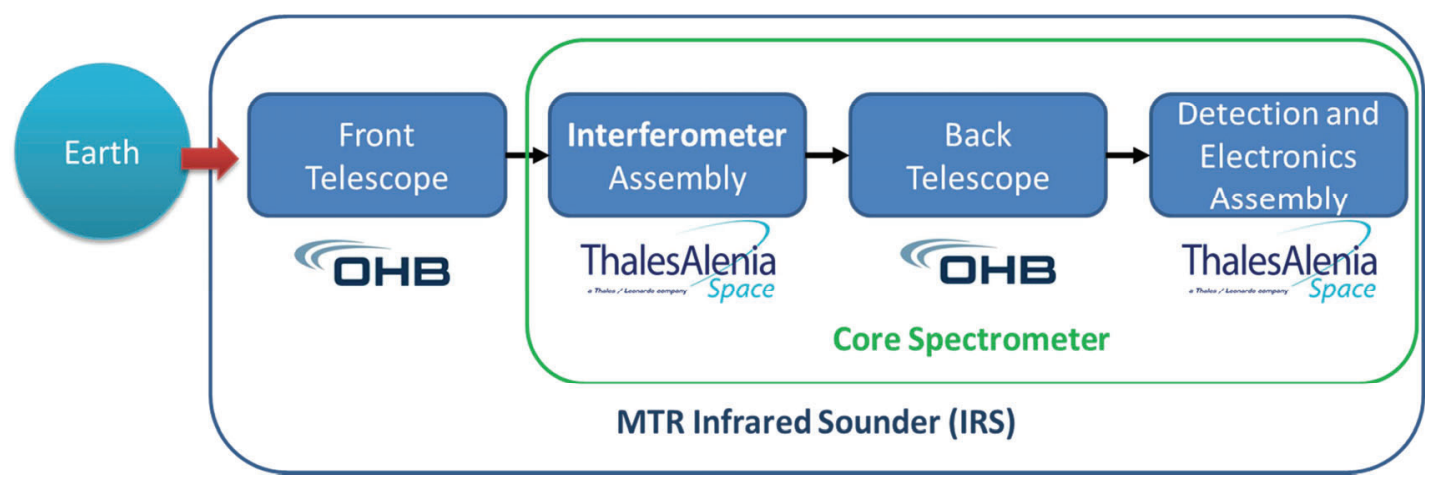

Figure 2: The MTG-IRS architecture is made of four main elements built by European manufacturers. The Core spectrometer is a subpart that is representative of the spectral behavior of the instrument.

\subsection{MTG-IRS spectral performances specifications overview}

The spectral performances are specified in the Metosat Third Generation system requirements document [4]. The main spectral performance requirements are:

- Spectral band and spectral resolution specifications [Table 1]

- Spectral Response Function (SRF) Knowledge and stability requirement [Table 2]. This point is important, as the SRF is the key to the correct interpretation of acquired spectrogram, as illustrated in Figure 3.

- $\quad$ Spectral lines/SRF positioning requirements [Table 3]

The spectral response function knowledge and stability requirements are particularly challenging. With the aim to fulfill this specification, Thales Alenia Space had to implement a new patented metrology system [8]. This system allows to know the 3D relative positioning of the two arms of the Michelson interferometer. It allows to get a subnanometric knowledge of Optical Path Difference (OPD) fluctuations for each sounding points (i.e. for each detector pixel) of the instrument field of view. The validation of this technology is the core topic of this paper.

Table 1: Spectral band and spectral resolution specifications [4]: MTG-IRS spectral bands definition:

\begin{tabular}{|l|l|l|l|l|}
\hline $\begin{array}{c}\text { Spectral } \\
\text { Band }\end{array}$ & \multicolumn{1}{|c|}{ Status } & $\begin{array}{c}\text { Wavenumber } \\
\text { range }\end{array}$ & $\begin{array}{c}\text { Spectral Channel } \\
\text { Interval } \Delta v\end{array}$ & $\begin{array}{c}\text { Spatial } \\
\text { Sampling } \\
\text { Distance(SSD) }\end{array}$ \\
\hline LWIR & Extended & $680-700 \mathrm{~cm}^{-1}$ & $\begin{array}{l}\text { Less than or equal } \\
\text { to } 0.625 \mathrm{~cm}^{-1}\end{array}$ & $4.0 \mathrm{~km}$ \\
\cline { 2 - 5 } & Specified & $700-1210 \mathrm{~cm}^{-1}$ & & \\
\hline MWIR & Specified & $1600-2175 \mathrm{~cm}^{-1}$ & $\begin{array}{l}\text { Less than or equal } \\
\text { to } 0.625 \mathrm{~cm}^{-1}\end{array}$ & $4.0 \mathrm{~km}$ \\
\cline { 2 - 3 } & Extended & $2175-2250 \mathrm{~cm}^{-1}$ & & \\
\hline
\end{tabular}

MTG-IRS spectral resolution requirement:

\section{PL-IRS-210-Level 1b}

The full width half maximum (FWHM) of the IRS spectral sample spectral response function (SRF) shall be less than or equal to $0.754 \mathrm{~cm}^{-1}$. 
Table 2: Spectral Response Function knowledge and stability specifications:

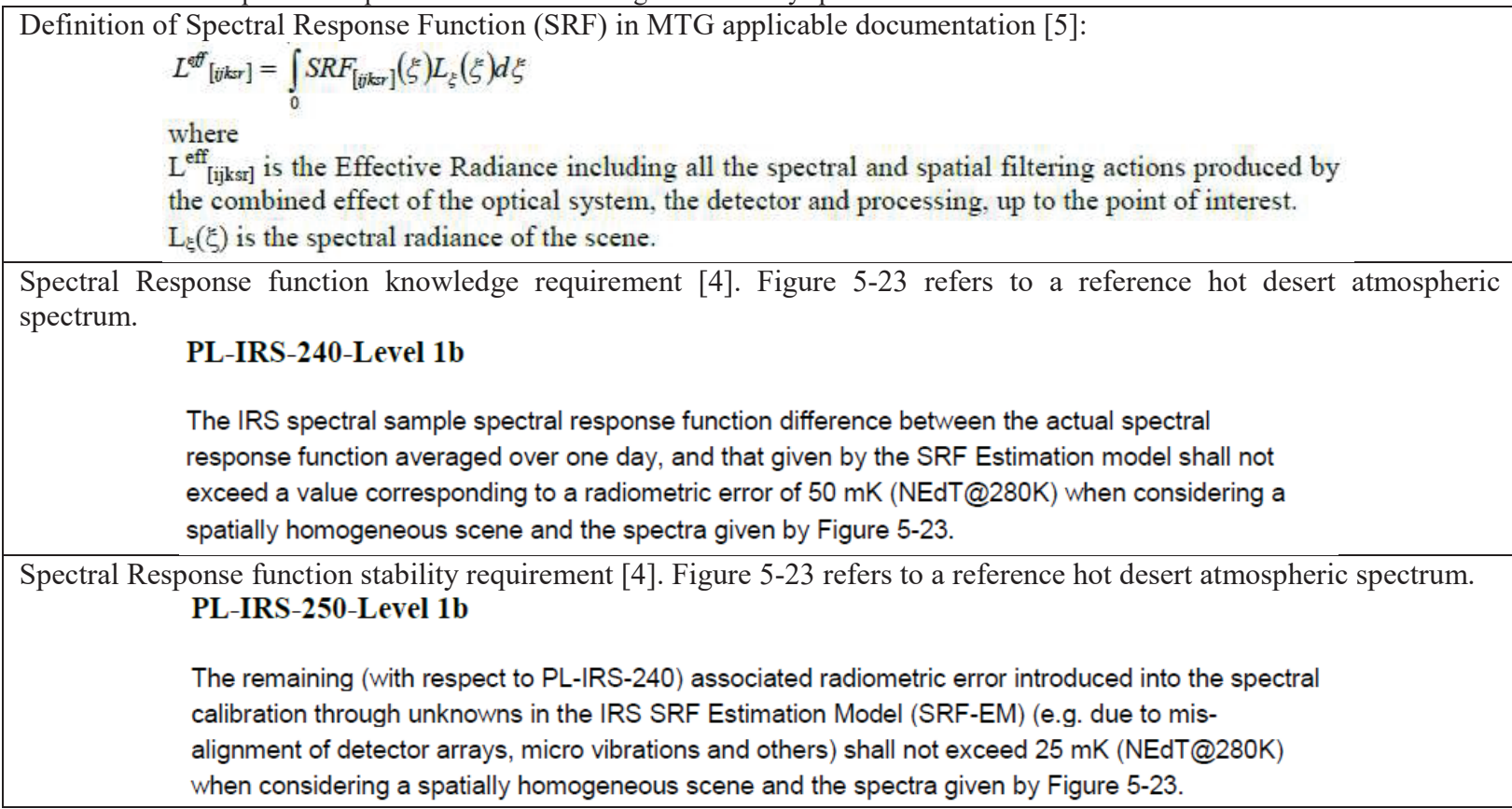

Table 3: Spectral lines/SRF positioning specifications [4]:

\section{MTG-IRS spectral bands definition:}

\section{PL-IRS-230-Level 1b}

The position of the IRS spectral sample SRF centroid wavenumber shall be determined by the spectral calibration algorithm and shall be stable over the spectral calibration period such that the relative wavenumber knowledge accuracy $\delta v / v$ does not exceed $3^{*} 10^{-6}$ in LWIR and $1^{*} 10^{-6}$ in MWIR.

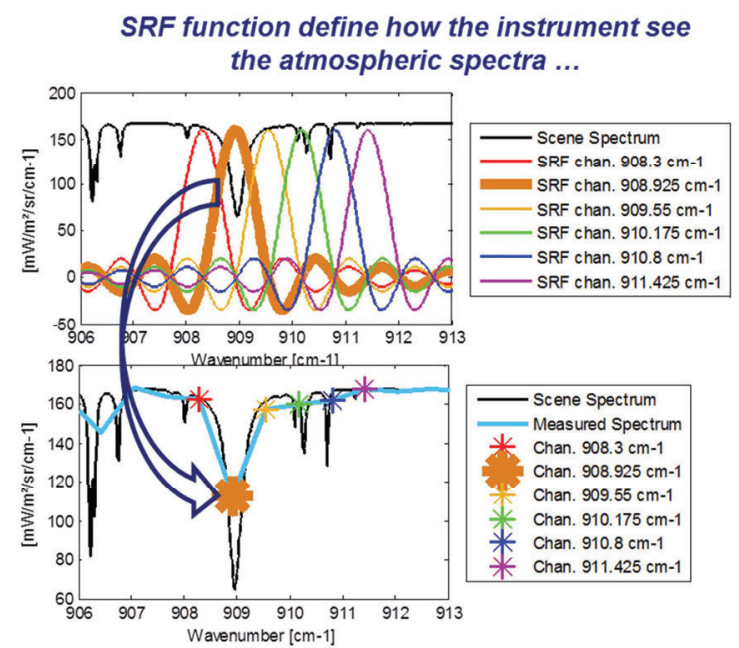

Figure 3: The rendering of a spectral line in the measured spectrum depends on the SRF shape, that's why it is important to have a nearly perfect knowledge of those functions. 


\section{MTG-IRS SPECTRAL PERFORMANCES VERIFICATION STRATEGY}

The development of the MTG-IRS is using Thales Alenia Space IASI instrument heritage and a pre-validation of metrology principles on an early development breadboard.

This heritage enables to build complex numerical simulators of the instrument, which were used to size the IRS interferometer and science data processing units. Those simulators are used to compute the expected flight performances of the instrument.

The goal of the testing campaign is to validate model predictions. These model predictions will be validated in several steps, as presented in the validation timeline (Figure 4):

1/ Validation of SRF function knowledge accuracy.

Validation of the SRF function knowledge (temporally averaged) on the core spectrometer, which is representative of the instrument in term of SRF.

The verification is performed by measuring the response of the instrument to a monochromatic line source. This response is named Instrument Line Shape (ILS) function. This function is similar to SRF function: ILS and SRF are often supposed to be equivalent (see MTG applicable document [5]). Indeed:

- $\mathrm{SRF}$ is the response of one specific spectral channel to each wavenumber.

- ILS is the spectrum measured (all spectral channels) for one specific input wavenumber.

The measured ILS is compared to the expected ILS shape function, which is computed by the MTG-IRS SRF estimation model algorithm.

Obtained results are presented in $\S 3$

2/ Validation of SRF function stability.

The SRF function stability performance is driven by the robustness of the instrument to mechanical perturbations (microvibrations). The sensitivity of the instrument to such perturbation necessitates to implement a 3D metrology system that allows to characterize the perturbations, and data correction algorithms to reduce those perturbations residual on performances to an acceptable level.

There are several difficulties to validate this robustness:

- The performance specification is stringent (the effect is one order of magnitude below radiometric noise performance requirements). The residual perturbations are hard to detect on test measurements.

- In the interferometer testing configuration, ambient micro-vibration environment is not representative of flight environment.

To cope with those two issues, the performance verification is held in three steps:

- On the Interferometer Assembly (IA, interferometer alone), high levels of mechanical perturbations are injected using shakers (§4.1). The perturbation shall be high enough to enable an easy detection of perturbation residual on acquired spectra. The measured residual error is compared with the residual predicted by numerical models. The matching of the two results allows to prove that the numerical simulation is mature and accurate enough to compute the performance residual for a given level of perturbation. The injection of high levels of mechanical perturbation allows to by-pass the error residual detectability issue. Results are detailed in $\S 4.2$.

- In order to assess the level of mechanical perturbation that will reach the interferometer in flight, a dedicated mechanical test is performed on the "Structural and Thermal Model" (a hardware model representative of MTG-IRS mechanical and thermal behavior). See $\S 4.2$.

- Once the numerical performance model and the expected perturbation levels are validated, the expected perturbation levels are injected into numerical performance model to assess and confirm the future flight performances. 


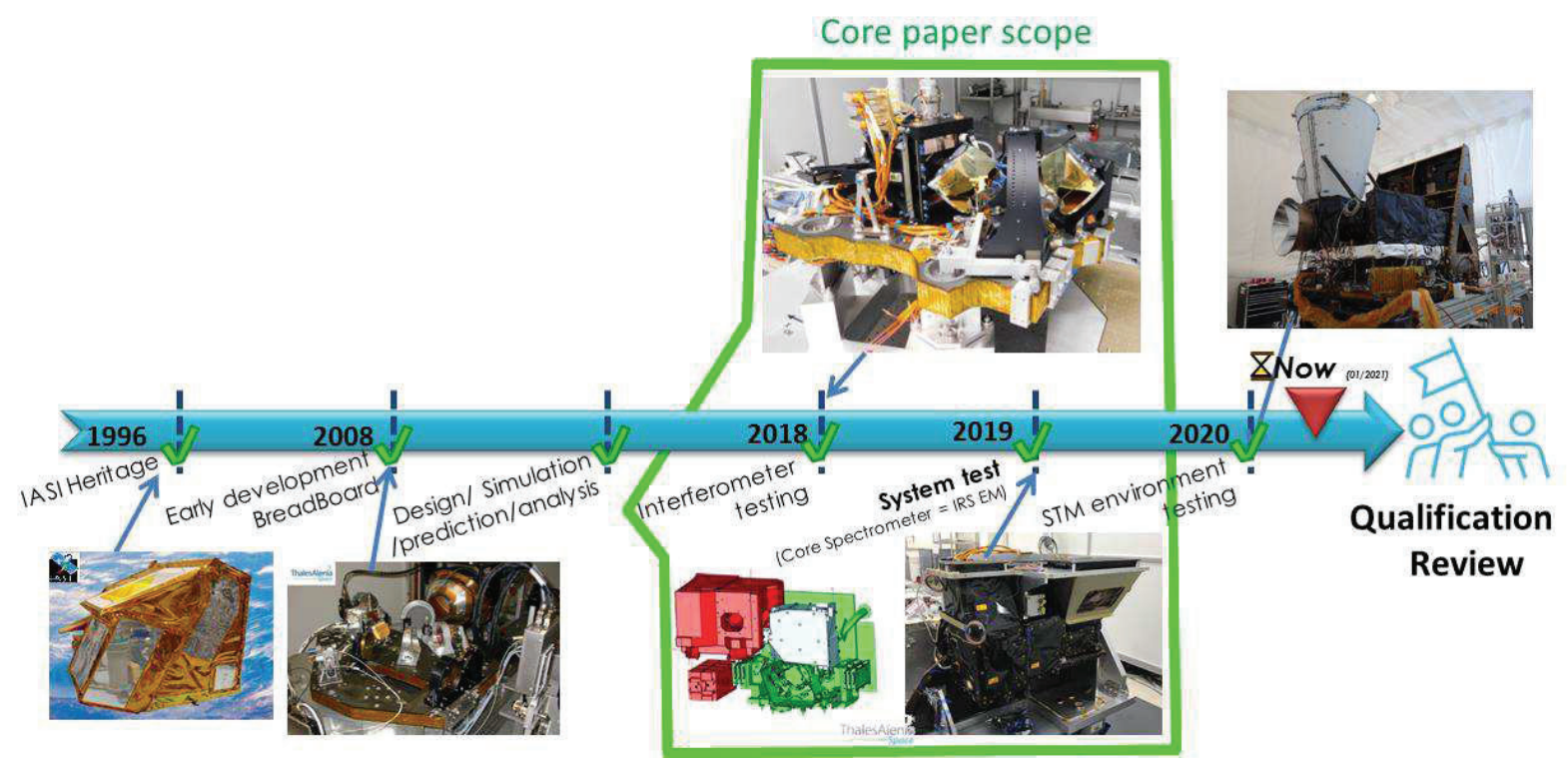

Figure 4: Spectral performance validation timeline to interferometer assembly qualification review.

\section{SPECTRAL PERFORMANCE TESTING: PART 1, INSTRUMENT LINE SHAPE KNOWLEDGE ACCURACY}

The instrument line shape knowledge was verified during the core spectrometer test campaign. The core spectrometer is the subpart of the MTG-IRS instrument (Figure 2 and Figure 5) which is representative of instrument spectral behavior.

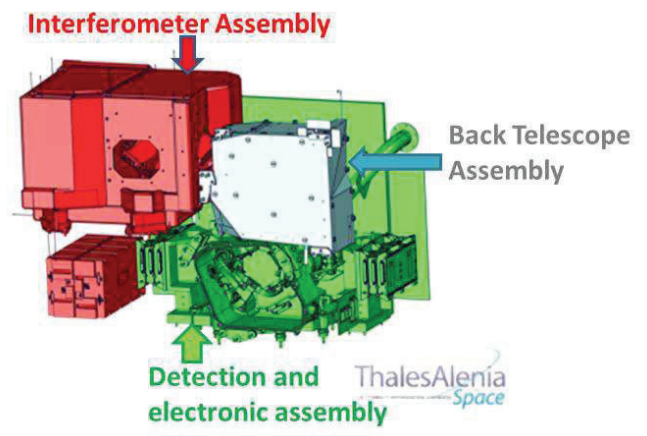

Figure 5: Core Spectrometer is composed of the Michelson Interferometer Assembly (IA, in red), the Back Telescope Assembly (BTA, in gray) and Detection and Electronics Assembly (DEA, in green).

\subsection{Test set-up: Core spectrometer illuminated by laser sources}

Test set-up is presented in Figure 6. The idea is to produce a spatially uniform monochromatic scene at core spectrometer entrance with the aim to measure the instrument line shape (ILS).

For this purpose, two laser beams (one for each spectral band) are injected into an integrating sphere placed at core spectrometer entrance. The test equipment needs to be carefully dimensioned, in particular:

- The wavenumber position is stabilized thanks to a control loop to maintain a relative stability better than $\sim 1 \mathrm{e}-6$. The line position is measured with a lambdameter to catch and follow the potential residual fluctuations. 
- The laser line width shall be much smaller than the targeted instrument spectral resolution with the aim to be able to assume the source as perfectly monochromatic.

- A speckle reduction system shall be implemented. The necessity of this kind of system for ILS measurement is documented by Simon Potvin and Jérôme Genest [6].

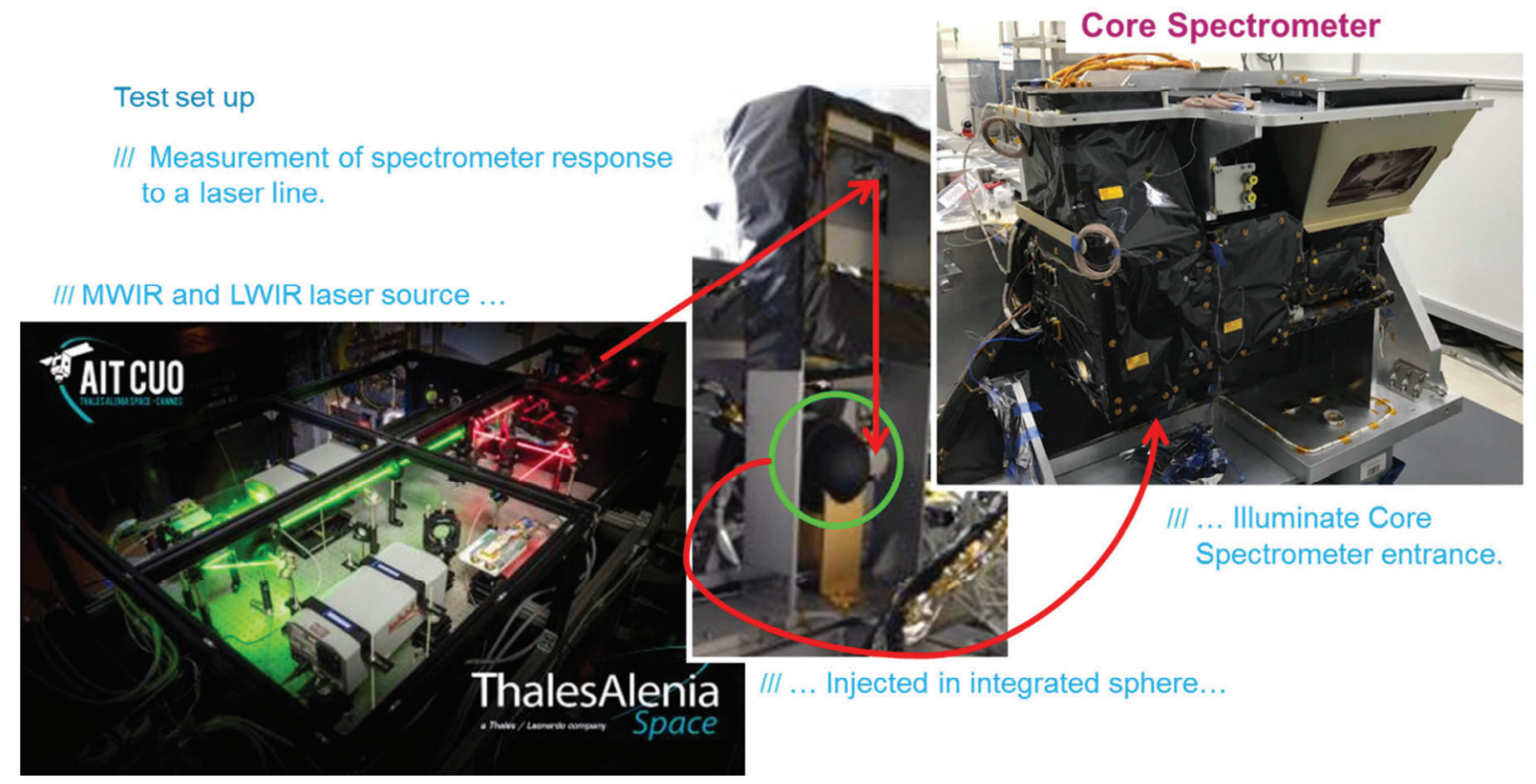

Figure 6: Core Spectrometer spectral test set-up: two MWIR and LWIR laser beams are injected in an integrating sphere to produce a spatially homogenous monochromatic scene to enlighten the Core spectrometer entrance. The integrating sphere and the core spectrometer being placed into a vacuum chamber. The laser is injected into the chamber through porthole.

\subsection{Comparison of measured line instrument line shape with prediction}

For each pixel of each spectral band, the ILS (core spectrometer response to the monochromatic light) is computed and compared to the expected shape. The obtained average ILS is, for each pixel, a temporal average of 236 ILS measurements. The result is illustrated in Figure 7.

The obtained match shall be compared with system specification (recalled in Table 2). For this purpose, the specification reference spectrum is convolved with the two ILS (measured and predicted) and the difference between the two results is expressed in radiometric unit (as defined in [5] => Noise Equivalent delta Temperature definition). The following results are obtained:

- $\quad$ MWIR $10 \mathrm{mK}$ to $20 \mathrm{mK}$

- $\quad$ LWIR: $20 \mathrm{mK}$ to $35 \mathrm{mK}$

Note 1: This result supposed that the ILS shape error does not depend on the wavelength, which is not rigorously exact.

This result is slightly better than expected and allows to demonstrate the quality of the SRF estimation model that will be used to provide the SRF information to MTG-IRS spectra end users. 

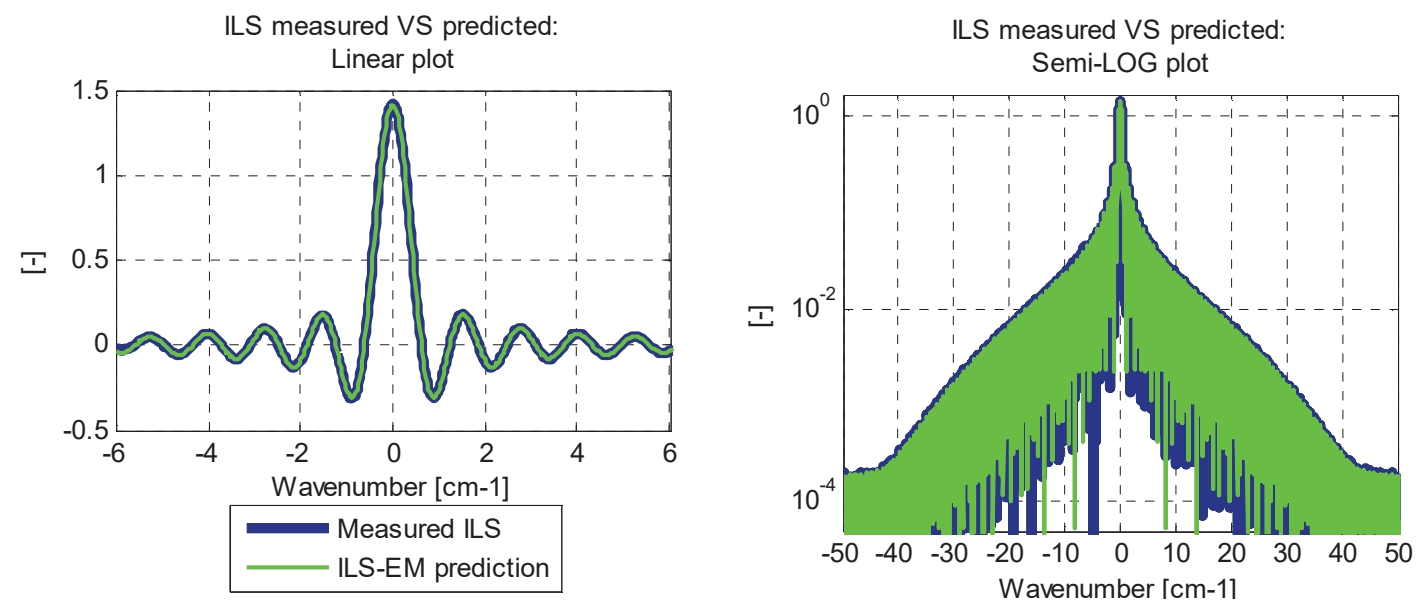

Figure 7: Comparison between measured core spectrometer ILS and prediction. The match appears to be visually nearly perfect. Remote side-lobes are perfectly described by the model even for lobes 10000 smaller than the main peak. The measurement curve on this plot is corresponding to the ILS measured on a MWIR pixel. It is an average of 236 ILS measurements.

\subsection{Line shape positioning stability results}

During Core Spectrometer test campaign, the stability of the ILS position was also checked.

The accurate spectral positioning of MTG-IRS spectra will be ensured thanks to a dedicated spectral calibration process. The spectral scale positioning will be checked regularly with the aim to perform the scale correction.

However the raw instrument positioning mismatch needs to be stable enough between two calibrations to ensure the quality of the correction.

This point was checked on core spectrometer. In LWIR, no ILS position instability was identified. In MWIR, a 1ppm fluctuation was identified (see Figure 8), but this fluctuation is corresponding to the real laser line position fluctuation, as measured by MWIR laser lambda-meter. Hence, this fluctuation is not due to the Core Spectometer, but to the test setup. The stability of the core spectrometer itself does not bring any significant instability.

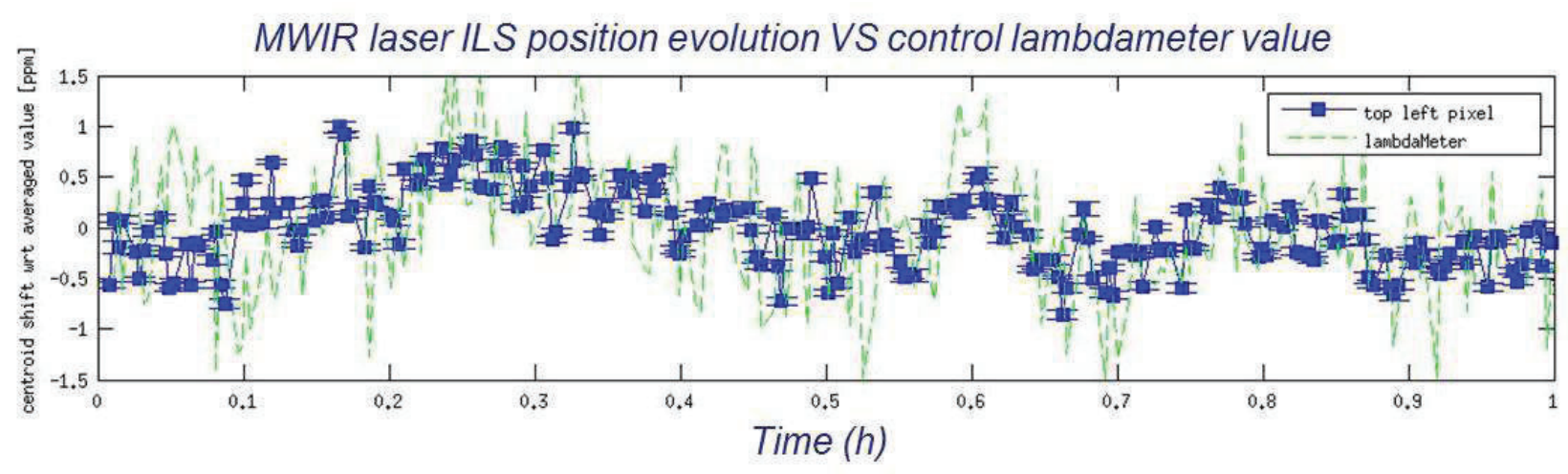

Figure 8: Measurement of Instrument Line Shape (ILS) position evolution during one hour in MWIR (blue) compared with laser source wavenumber stability measured by a lambdameter (green). The two curves are matching. No line positioning instability due to the core spectrometer is detected. 


\section{SPECTRAL PERFORMANCE TESTING: PART 2, 3D METROLOGY SYSTEM ROBUSTNESS TO MICRO-VIBRATION TEST.}

As explained in $\S 2$, the validation of the new 3D metrology system is performed in 3 steps:

- Validation of vibration robustness prediction numerical model during a test on the Interferometer Assembly (IA)

- Assessment of the flight vibration perturbation levels by testing the MTG-IRS Structural and Thermal Model (STM)

- Computation of the flight performance using validated numerical model and the micro vibration perturbation measured during STM test.

\subsection{Set-up for interferometer assembly micro-vibration sensitivity test}

As for core spectrometer test, the Interferometer Assembly is illuminated with a spatially uniform monochromatic scene produced by a laser injected in an integrating sphere. The interferometer output signal is collected by a ground optical system that simulates IRS back optics (Back Telescope Assembly and Detection and Electronics Assembly) with the aim to measure ILS.

The Interferometer is placed on a heavy structure that is voluntarily vibrated using a shaker (Figure 9).

Acquired interferograms are processed using the same algorithm as the ones that are implemented in the flight data processing unit.

Residual error due to the injected perturbation is identified, measured, and compared to the result predicted using numerical model simulations.

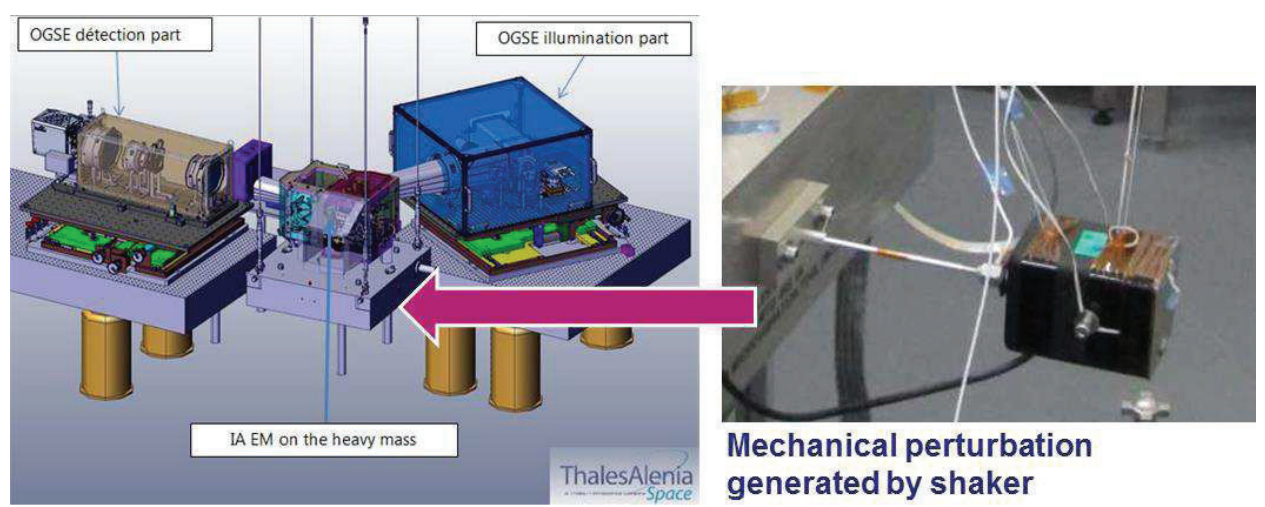

Figure 9: Interferometer Assembly sensitivity to vibration test set-up. OGSE = Optical Ground Support Equipment.

\subsection{Micro-vibration sensitivity test results}

Thanks to the high level of injected perturbation, a vibration residual error is visible on ILS measurement. The residual can be unambiguously identified as it is a ghost (repetition of the ILS peak shape) which is located at a predictable distance from the ILS main peak:

$$
\text { ghost_position }=\frac{f \_p e r t u r b a t i o n}{\text { opd_speed }}
$$

... where f_perturbation is the injected perturbation frequency and opd_speed is the nominal Optical Path Difference scanning speed of the interferometer. 
Figure 10 illustrates the result for the $22 \mathrm{~Hz}$ perturbation frequency: The residual ghost is detected at the expected location. Its amplitude is measured (1.01e-3 with respect to main ILS peak) and compared to the expected level of residual ghost amplitude assessed using numerical simulation models (0.94-3 with respect to main ILS peak).

The good match between measurement and prediction allows to validate the accuracy of the model to predict performance error residual knowing the mechanical perturbation level.

It is now possible to rely on this numerical model to compute flight performances using estimations of flight mechanical perturbation levels.

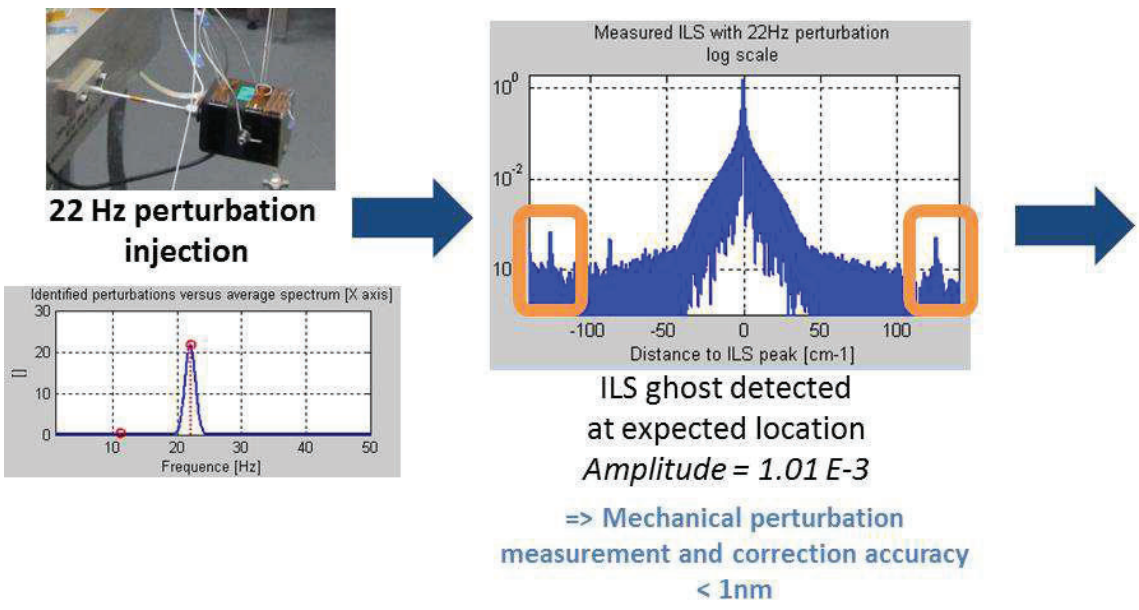

\section{Comparison with predicted residual:}

Measured Amplitude: $1.01 E-3$

Performance model prediction: $0.94 E-3$

\section{Performance model} validated!

Figure 10: Interferometer Assembly sensitivity to vibration test result for $22 \mathrm{~Hz}$ perturbation. OGSE = Optical Ground Support Equipment.

\subsection{Overview of structural an thermal model micro-vibration testing}

The MTG-IRS Structural and Thermal Model (STM) is a non-functional engineering model of the MTG-IRS instrument. It is only representative in terms of mechanical and thermal behavior. It is used to test and validate thermal and mechanical behavior.

The STM test campaign includes a dedicated test to measure perturbation that reach the interferometer and that produce perturbations on the Optical Path Difference scanning. With the aim to do this test, the Interferometer Assembly STM was removed from the IRS STM. It was replaced by a functional Engineering Model of the Interferometer Assembly. The 3D metrology of the interferometer was then used as a mechanical vibration sensor.

The test provided information about the mechanical perturbation levels induced by instruments equipment such as scanning mirrors or the cryo-cooler.

The main perturbations come from the cryo-cooler. The cryo-cooler frequency and its harmonics were identified thanks to the Interferometer 3D metrology system. Those perturbation levels are lower than predicted by mechanical simulations used up to now to assess future MTG-IRS flight performances.

This test also demonstrates the ability of the metrology system to detect sub-nanometric perturbations: the smallest detected perturbations have an estimated amplitude of $0.03 \mathrm{~nm}$ for harmonic 13 of the cryo-cooler fundamental frequency. 


\subsection{Synthesis of 3D metrology performances.}

The results of interferometer micro-vibration sensitivity tests $(\S 4.2)$ allows to validate the numerical model used for performance prediction. The micro-vibration test held on Structural and Thermal model of the instrument (§4.3) allows to confirm that the micro-vibration perturbation hypothesis used during interferometer development to assess performances are conservative.

Hence, we can conclude that the previous global performance predictions of the spectral noise due to vibrations are valid. It confirms the validity of the new 3D metrology solution used on MTG-IRS.

This result will be used for the qualification review of the interferometer assembly that is expected in 2021.

\section{CONCLUSION}

Thanks to three successful test campaigns held at three different levels (Interferometer Assembly, Core Spectrometer and full instrument Structural and Thermal Model), the demonstration was made that the spectral behavior of as deigned Metosat Third Generation Infrared Sounder is as expected, in particular:

- $\quad$ Spectral Response Function shape is known with a sufficient level of accuracy (as demonstrated in $\S 3$ )

- $\quad$ The efficiency of the new 3D metrology system implemented in the Interferometer is demonstrated (see $\S 4$ ).

Those results are decisive for the qualification of the interferometer which is expected in 2021, and allow to confirm the adequacy of the selected design.

\section{REFERENCES}

[1] A. A. Michelson, Philos. Mag. 31, 256 (1891)

[2] A. A. Michelson, Light Waves and Their Uses, University of Chicago Press, Chicago, 1902 (reissued in paperback, Pheonix Edition, 1961).

[3] Peter R. Griffiths, James A. de Haseth., [Fourier Transfor Infrared Spectrometry, Second Edition], A Jhon Wiley \& sons, inc., publication (2007).

[4] European Space Agency, "Meteosat Third Generation (MTG) SpaceCraft System Requirements Document (SC_SRD) [AD03]”, ref. MTG.ESA.SA.RS.0062 issue 3.3 (23 October 2013)

[5] European Space Agency, "Meteosat Third Generation (MTG) Terms, Definitions, Conventions and Abbreviations [AD16]", ref. MTG.ESA.SA.RS.0052 issue 4.1 (02 September 2013)

[6] Potvin S, Genest J. Reducing the effect of integrating sphere speckle when characterizing the instrument line shape of a Fourier-transform hyperspectral imager. Appl Opt. 2009 Oct 20;48(30):5849-52. doi: 10.1364/AO.48.005849. PMID: 19844324.

[7] Fabienne Casoli, , Carole Deniel, Cathy Clerbaux, Adrien Deschamps, Cyril Crevoisier and Pierre Tabary. « IASI: summary of a success story and perspectives ». 4th IASI Conference (11-15 april 2016, Juan-Les-Pins, France)

[8] Patent reference: FR2972797 SYSTEME ET PROCEDE DE METROLOGIE APPLIQUE A UN INTERFEROMETRE D'ANALYSE A DISTANCE D'UN COMPOSE. 